\title{
COMBINATORIAL FORMULAE FOR THE $\chi_{y}$-GENUS OF A MULTIORIENTED QUASITORIC MANIFOLD
}

\author{
T. E. PANOV
}

In [1] the authors introduced the class of smooth manifolds with a compact torus action whose orbit space carries combinatorial structure of a simple polytope. Following [2], [3], we call these manifolds quasitoric. The name refers to the fact that topological and combinatorial properties of quasitoric manifolds are similar to that of non-singular algebraic toric varieties [4] (or toric manifolds). Any quasitoric manifold is defined by combinatorial data: the lattice of faces of a simple polytope and a characteristic function that assigns an integer primitive vector defined up to sign to each facet. Despite their simple and specific definition, quasitoric manifolds in many cases may serve as model examples (for instance, each complex cobordism class contains a quasitoric manifold [5]). All these facts enable to use quasitoric manifolds for solving topological problems by combinatorial methods and vice versa. Some applications were obtained in [2], [6], where quasitoric manifolds are studied in the general context of "manifolds defined by simple polytopes". Unlike toric varieties, quasitoric manifolds may fail to be complex; however, they always admit a stably (or weakly almost) complex structure. As it was shown in [3], a stably complex structure (i.e. a complex structure in the stable tangent bundle) on a quasitoric manifold is also defined combinatorially, namely, by specifying an orientation of the simple polytope and choosing signs for vectors given by the characteristic function. A quasitoric manifold with such additional structure was called in [3] multioriented. Hence, a multioriented quasitoric manifold determines a complex cobordism class, for which one can define characteristic numbers and complex Hirzebruch genera. We calculate the $\chi_{y}$-genus (in particular, the signature and the Todd genus) in terms of the combinatorial data by applying the Atiyah-Hirzebruch formula to one specific circle action with isolated fixed points.

A convex $n$-dimensional polytope $P^{n}$ is called simple if the number of codimension-one faces (or facets) meeting at each vertex is $n$. Any simple polytope is a manifold with corners. Let $M^{2 n}$ be a compact $2 n$-dimensional manifold with an action of the torus $T^{n}=\left\{\left(e^{2 \pi i \varphi_{1}}, \ldots, e^{2 \pi i \varphi_{n}}\right) \in \mathbb{C}^{n}\right\}, \varphi_{i} \in \mathbb{R}$. Then $M^{2 n}$ is called a quasitoric manifold if the $T^{n}$-action is locally isomorphic to the standard action of $T^{n}$ on $\mathbb{C}^{n}$ by diagonal matrices, while the orbit space is diffeomorphic, as manifold with corners, to a simple polytope $P^{n}$ (see [1], [3] for the details).

Let $M^{2 n}$ be a quasitoric manifold with orbit space $P^{n}$, and let $\mathcal{F}=\left\{F_{1}, \ldots, F_{m}\right\}$ be the set of facets of $P^{n}, m=\sharp \mathcal{F}$. The interior of facet $F_{i}$ consists of orbits with the same one-dimensional isotropy subgroup $G_{F_{i}}=\left\{\left(e^{2 \pi i \lambda_{1 i} \varphi}, \ldots, e^{2 \pi i \lambda_{n i} \varphi}\right) \in T^{n}\right\}$, $\varphi \in \mathbb{R}$. In this way one defines a characteristic function $\lambda: \mathcal{F} \rightarrow \mathbb{Z}^{n}$ that takes facet $F_{i}$ to the primitive vector $\lambda_{i}=\left(\lambda_{1 i}, \ldots, \lambda_{n i}\right)^{\top} \in \mathbb{Z}^{n}$ (which is defined only up to sign), and a $(n \times m)$-matrix $\Lambda$ with columns $\lambda\left(F_{i}\right)$. Each vertex $p$ of $P^{n}$ can be represented as the intersection of $n$ facets: $p=F_{i_{1}} \cap \cdots \cap F_{i_{n}}$; we denote by $\Lambda_{(p)}=\Lambda_{\left(i_{1}, \ldots, i_{n}\right)}$ the minor matrix of $\Lambda$ formed by the columns $i_{1}, \ldots, i_{n}$. Then $\operatorname{det} \Lambda_{(p)}=\operatorname{det}\left(\lambda_{i_{1}}, \ldots, \lambda_{i_{n}}\right)= \pm 1$. In what follows we refer to the vectors $\lambda_{i}=\lambda\left(F_{i}\right)$ as facet vectors.

This work was partly supported by the Russian Foundation for Fundamental Research (grant no. 99-01-00090). 
We suppose that the torus $T^{n}$ is oriented; then an orientation of $M^{2 n}$ is specified by orienting the polytope $P^{n}$ and vice versa. The inverse image $\pi^{-1}\left(F_{i}\right)$ of the facet $F_{i}$ under the orbit map $\pi: M^{2 n} \rightarrow P^{n}$ is a quasitoric submanifold $M_{i} \subset M^{2 n}$ of codimension 2. A choice of sign for the vector $\lambda\left(F_{i}\right)$ is equivalent to a choice of orientation (or stably complex structure) of the normal bundle $\nu_{i}:=\nu\left(M_{i} \subset M^{2 n}\right)$. This orientation in turn is uniquely determined by orientations of $M^{2 n}$ and $M_{i}$. An oriented quasitoric manifold $M^{2 n}$ with fixed orientations of facial submanifolds $M_{i}=\pi^{-1}\left(F_{i}\right)$ was called in [3] multioriented. The oriented submanifold $M_{i} \subset M^{2 n}$ of codimension 2 gives rise, by the standard topological construction, to a complex line bundle $\sigma_{i}$ over $M^{2 n}$ that restricts to $\nu_{i}$ over $M_{i}$. The following theorem from [3] shows that a multioriented quasitoric manifold can be invested with a canonical stably complex structure.

Theorem 1. The following isomorphism of real oriented $2 m$-bundles holds for any quasitoric manifold $M^{2 n}$ :

$$
\tau\left(M^{2 n}\right) \oplus \mathbb{R}^{2(m-n)} \simeq \sigma_{1} \oplus \cdots \oplus \sigma_{m} .
$$

Here $\tau\left(M^{2 n}\right)$ is the tangent bundle and $\mathbb{R}^{2(m-n)}$ denotes the trivial $2(m-n)$-bundle over $M^{2 n}$.

Thus, an oriented simple polytope $P^{n}$ and characteristic matrix $\Lambda$ not only define a (multioriented) quasitoric manifold, but also specify its cobordism class in the complex cobordism ring $\Omega_{U}$.

The interior of an edge (one-dimensional face) of $P^{n}$ consists of orbits with the same $(n-1)$-dimensional isotropy subgroup $G_{E_{j}}=\left\{\left(e^{2 \pi i \varphi_{1}}, \ldots, e^{2 \pi i \varphi_{n}}\right) \in\right.$ $\left.T^{n}: \mu_{1 j} \varphi_{1}+\ldots+\mu_{n j} \varphi_{n}=0\right\}$. This subgroup is defined by a primitive vector $\mu_{j}=\left(\mu_{1 j}, \ldots, \mu_{n j}\right)^{\top}$. We refer to this $\mu_{j}$ as edge vector; again it is defined only up to sign. If $E_{j_{1}}, \ldots, E_{j_{n}}$ are edges that meet at a vertex $p$ and $\mathrm{M}_{(p)}$ is the matrix with columns $\mu_{j_{1}}, \ldots, \mu_{j_{n}}$, then $\operatorname{det} \mathrm{M}_{(p)}=\operatorname{det}\left(\mu_{j_{1}}, \ldots, \mu_{j_{n}}\right)= \pm 1$.

The next lemma enables to choose signs of edge vectors for a multioriented quasitoric manifold unambiguously "locally" at each vertex.

Lemma 2. Signs for the edge vectors $\mu_{j_{1}}, \ldots, \mu_{j_{n}}$ meeting at $p$ can be chosen in such a way that $\mathrm{M}_{(p)}^{\top} \cdot \Lambda_{(p)}=E$ (unit matrix).

The orientation of $P^{n}$ defines an ordering of edges at each vertex, which in turn fixes an ordering of the corresponding edge vectors.

Definition 3. The sign of a vertex $p \in P^{n}$ is

$$
\sigma(p)=\operatorname{det} \mathrm{M}_{(p)}=\operatorname{det}\left(\mu_{i_{1}}, \ldots, \mu_{i_{n}}\right),
$$

where $\mu_{i_{1}}, \ldots, \mu_{i_{n}}$ are canonically ordered edge vectors meeting at $p$.

Theorem 4. Suppose that $\nu \in \mathbb{Z}^{n}$ is an integer primitive vector such that $\left\langle\mu_{i}, \nu\right\rangle \neq$ 0 for all edge vectors $\mu_{i}$. Then the circle subgroup $S^{1} \subset T^{n}$ defined by $\nu$ acts on $M^{2 n}$ with isolated fixed points corresponding to vertices of $P^{n}$. In the tangent space $T_{p} M^{2 n}$ at fixed point corresponding to the vertex $p=F_{i_{1}} \cap \cdots \cap F_{i_{n}}$ this action induces the representation of $S^{1}$ with weights $\left\langle\mu_{i_{1}}, \nu\right\rangle, \ldots,\left\langle\mu_{i_{n}}, \nu\right\rangle$.

Definition 5. Given the $S^{1}$-action on $M^{2 n}$ defined by a primitive vector $\nu$, the index of the vertex $p=F_{i_{1}} \cap \cdots \cap F_{i_{n}}$ is

$$
\operatorname{ind}_{\nu}(p)=\left\{\sharp k:\left\langle\mu_{i_{k}}, \nu\right\rangle<0\right\},
$$

i.e. $\operatorname{ind}_{\nu}(p)$ equals the number of negative weights at $p$. 
Theorem 6. Let $M^{2 n}$ be a multioriented quasitoric manifold, and let $\nu$ be a vector described in Theorem 4. Then

$$
\chi_{y}\left(M^{2 n}\right)=\sum_{p \in P^{n}}(-y)^{\operatorname{ind}_{\nu}(p)} \sigma(p),
$$

where the sum is taken over all vertices of $P^{n}$.

The idea of proof of this theorem is to apply the Atiyah-Hirzebruch formula [7] for the $\chi_{y}$-genus to $S^{1}$-action on $M^{2 n}$ from Theorem 4 .

As particular cases of $\chi_{y}$-genus one obtains the $n$th Chern number $c_{n}\left[M^{2 n}\right]$ $(y=-1)$, the signature $(y=1)$, and the Todd genus $(y=0)$. In the case of $n$th Chern number Theorem 6 gives

$$
c_{n}\left[M^{2 n}\right]=\sum_{p \in P^{n}} \sigma(p) .
$$

If $M^{2 n}$ is a complex manifold (e.g., $M^{2 n}$ is a smooth toric variety) one has $\sigma(p)=1$ for all vertices $p \in P^{n}$ and $c_{n}\left[M^{2 n}\right]$ equals the Euler number $e\left(M^{2 n}\right)$. In the general case the Euler number is also equal to the number of vertices of $P^{n}$, however this number may be different from $c_{n}\left[M^{2 n}\right]$.

The formula for the signature that is obtained from Theorem 6 can be rewritten in a form that is independent of a stably complex structure (i.e. of a multiorientation) and is determined only by an orientation of $M^{2 n}$. This reflects the fact that the signature is a homotopy invariant of an oriented manifold.

For the Todd genus, the formula from Theorem 6 is undefined. Additional analysis shows that the following theorem holds.

Theorem 7. The Todd genus of a multioriented quasitoric manifold can be calculated as

$$
\operatorname{td}\left(M^{2 n}\right)=\sum_{p \in P^{n}: \operatorname{ind}_{\nu}(p)=0} \sigma(p)
$$

(the sum is taken over all vertices of index 0$)$.

The author is grateful to Professor V. M. Buchstaber for stimulating discussions and useful recommendations.

\section{BIBLIOGRAPHY}

[1] M. Davis and T. Januszkiewicz, Duke Math. J. 62 (1991), no. 2, 417-451.

[2] V. M. Buchstaber and T.E. Panov, Trudy Matematicheskogo Instituta imeni V. A. Steklova 225 (1999), 96-131; English transl. in: Proceedings of the Steklov Institute of Mathematics 225 (1999), 87-120.

[3] V.M. Buchstaber and N. Ray, Tangential structures on toric manifolds, and connected sums of polytopes, preprint UMIST, Manchester, 1999.

[4] V. I. Danilov, Uspekhi Mat. Nauk 33 (1978), no. 2, 85-134; English transl. in: Russian Math. Surveys 33 (1978), 97-154.

[5] V.M. Bukhshtaber and N. Ray, Uspekhi Mat. Nauk 53 (1998), no. 2; English transl. in: Russian Math. Surveys 53 (1998), no. 2.

[6] V.M. Bukhshtaber and T.E. Panov, Uspekhi Mat. Nauk 53 (1998), no. 3, 195-196; English transl. in: Russian Math. Surveys 53 (1998), no. 3, 623-625.

[7] F. Hirzebruch, T. Berger, and R. Jung. Manifolds and Modular Forms, Second Edition, A Publication from the Max-Planc-Institut für Mathematik, Bonn, 1994.

Moscow State University

E-mail address: tpanov@mech.math.msu.su 